\title{
An Overview of the High Power Radio-Frequency Distribution System of the ESS
}

\author{
Norman Turner*t \\ University of Huddersfield \\ E-mail: U1474030@unimail.hud.ac.uk
}

The European Spallation Source (ESS) is a high brightness source of slow neutrons which is currently under construction in Sweden. ESS uses a linear accelerator to accelerate protons to 2000MeV. The superconducting cavities of the accelerator are supplied with power by a radiofrequency (RF) distribution system. The design, construction and operation of this RF system will provide valuable design information for an ADS driven by a linear accelerator. This paper provides an overview of the RF distribution system for ESS including protection against electrical breakdown in the waveguides.

4th Workshop on ADS and thorium

31 August - 2 September 2016

University of Huddersfield, England

* Speaker.

${ }^{\dagger}$ A footnote may follow. 


\section{Introduction}

The European Spallation Source (ESS) shares many significant features with proposed designs for accelerator driven subcritical reactors. In particular the ESS incorporates a high current superconducting linear accelerator as do designs proposed for the MYRRHA ADS [1] and the Chinese ADS systems [2]. The construction of the ESS involves a multinational collaboration which includes the UK [3]. As part of the collaboration the UK is making several in kind contributions to the ESS linac, the second largest being the high power RF distribution system for the superconducting cavities. This contribution is being provided by the University of Huddersfield in association with the UK's Science and Technology Facilities Council.

This paper provides an overview of the high power RF distribution system and then describes two aspects of the distribution system that have attracted significant attention, cooling and arc detection.

\section{ESS linac}

The ESS linac is illustrated in Figure 1. The duration of proton beam pulses will be $2.86 \mathrm{~ms}$ and the associated RF pulses to the cavities will have a duration of $3.5 \mathrm{~ms}$. Two types of cavity will be used: spoke cavities at $352.21 \mathrm{MHz}$ for proton energies up to $220 \mathrm{MeV}$ and elliptical cavities at 704.42 MHz for proton energies above 220 MeV. The linac will be housed in a tunnel over $400 \mathrm{~m}$ long. A gallery to house the RF sources will run parallel to much of the tunnel. Between the gallery and the tunnel there will be a series of stubs which provide conduits for RF power and ancillary cabling from the gallery to the cavities in the tunnel

\section{ESS RF Distribution}

The ESS requirement for 95\% reliability (availability) has strongly influenced design choices including those for the distribution of radio-frequency (RF) power to the cavities. In particular the ESS reliability target requires that the accelerator should run, after re-tuning, even when multiple RF sources or cavities are inoperable. To help achieve this each superconducting cavity will have a dedicated RF power source and a dedicated waveguide run. As there are 146 superconducting cavities large numbers of RF components are required and over $3 \mathrm{~km}$ of waveguides.

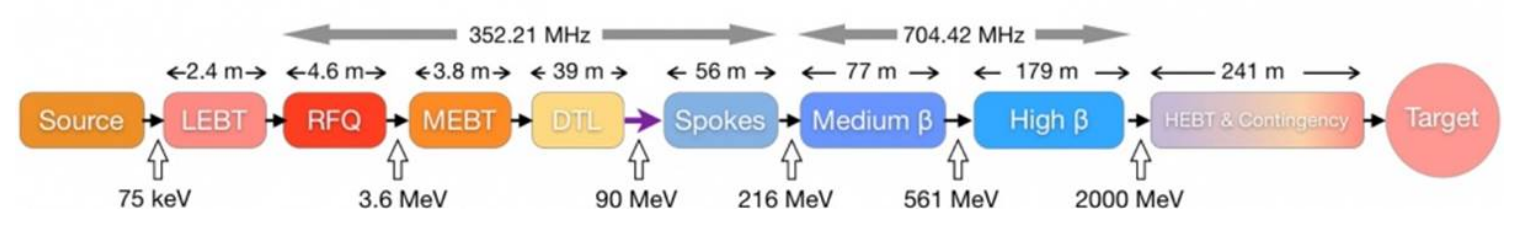

Figure 1: ESS Linac. Credit : ESS 
Figure 2 illustrates the RF distribution for a typical medium energy cavity. During filling of the cavities, RF power will be reflected back towards the relevant RF source. In order to protect the RF source from these reflections each waveguide run will incorporate a circulator which will divert any reflected power into a load. The loads will cooled by water. Each waveguide run will also incorporate directional couplers and multiple arc detectors. The directional couplers will be used to measure the forward and reflected power. This information is used by the LLRF (Low Level RF) subsystem, which regulates cavity tuning and sets the amplitude and phase for the RF sources.

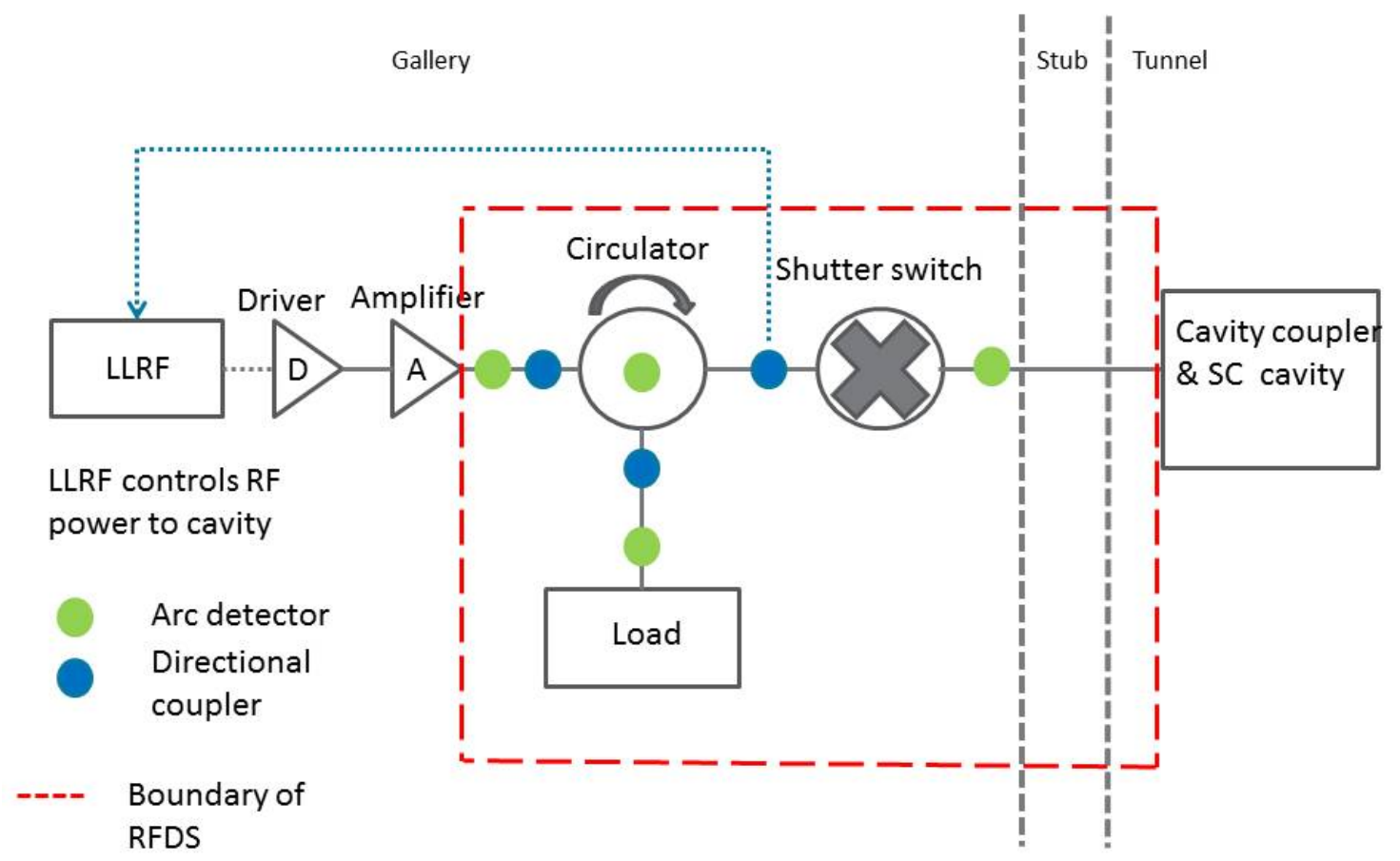

Figure 2: RFDS for Medium Beta Cavity

The waveguides will be air-filled, constructed from aluminium and operate in $\mathrm{TE}_{10}$ mode. The waveguide sizes will be WR1150 (704.42MHz) and half height WR2230 (352.21MHz). These sizes are sufficient to ensure that in normal circumstances the breakdown strength of air in the waveguide is not exceeded, even during periods of $100 \%$ reflection from the cavity. The internal surface finish of the waveguides will be to N6 or better. This corresponds to an average roughness of better than 0.8 micrometre which is small compared to the skin depth of aluminium (approximately 3.1 micrometre at $700 \mathrm{MHz}$ ). 


\section{Waveguide Cooling}

The waveguides are bundled into groups (usually 8) to transit the stubs. The power dissipated by a group of 8 waveguides in the stub is typically $>4 \mathrm{~kW}$ and the volume of the stubs is small, typically $1.2 \mathrm{~m} * 1.5 \mathrm{~m}$. The stubs will be sealed so there is very limited free convection and, without forced cooling, temperatures in stubs would be unacceptable high at $>200 \mathrm{C}$. The options considered to deliver forced cooling include water cooling from pipes mounted on the waveguides and internal forced air cooling (i.e. using the waveguides like HVAC ducts).

The water cooling option has several potential drawbacks. For example, brazing could distort the waveguide and inclusion of pipes in the stub would lead to further congestion in the already congested stub.

Forced internal air cooling is relatively novel although it was considered for the proposed APT accelerator [4]. It also has potential drawbacks. High air flow rates may be required to achieve satisfactory cooling, air filtering etc. will be required to reduce ingestion of particulates and there will be an need for additional fixtures in waveguide walls for air inlet and air outlet. Forced internal air cooling could also lead to tunnel air being released in the gallery. To address the potential issue of air release there may be a need to install RF windows in the waveguides. The RF windows would provide a barrier to the flow of air ingested from the tunnel, but their presence may impact on arc generation and detection. However forced internal air cooling may have an adventitious benefit because the likelihood of sustained arcs may be reduced in areas of high air flow.

\section{Arcs and Arc Detection}

Electrical breakdown of the air in a waveguide will reduce the RF power flowing to a cavity, reflect power back to the RF source and potentially damage waveguide walls and fixtures, especially RF windows [5]. ESS has identified that arcs with energies over $5 \mathrm{~J}$ have the potential to cause significant damage.

The electrical breakdown strength of a gas like air is dependent on $\frac{E}{p}$, where $E$ is the electric field and $p$ the pressure [6]. The electric field strength in waveguides can be enhanced by the presence of sharp conducting items or electrically conducting dust particles. In the latter case the enhancement can be particularly large if there is small inter-particle separation [7]. For high average levels of RF power heating of dust particles also increases the likelihood of electrical breakdown by locally reducing gas density.

General techniques for detecting arcs in waveguides include sensing RF power reflected by the arc and sensing optical emission from the arc [8], [9]. During cavity filling there is significant reflected RF power so simple detection of reflected RF power is an insufficiently refined technique for arc detection at ESS. Instead optical detectors will be used. 
ESS has a general policy of limiting equipment in the tunnel. For the RFDS this means that no arc detectors or fibre optics are allowed in the tunnel. The technical specifications of optical arc detectors typically include the threshold photometric illumination and the associated delay in response. To match the detection capability of the sensors to the size of arcs requires knowledge of both the efficiency of the arc in producing light (watts input to an arc per lumen emitted) and the losses for light as it propagates along the wave guide to the sensor. While there is extensive literature on electrical breakdown of air, the measurement of UHF arc luminosity as a function of absorbed energy does not seem to have attracted attention. To determine the losses as light from an arc propagates along a waveguide requires knowledge of both the waveguide geometry and the surface reflectivity. The simple specification of waveguide surface roughness by category (in this case N6) or average roughness (Ra) is insufficient to determine surface reflectivity for a ray striking at an arbitrary angle [10] .

\section{Conclusion}

The technology for RF power distribution is generally considered to be mature. However the combination of constraints at the ESS has created some novel challenges for the high power RF distribution system. Parts of the RF distribution system may also be required to act as a light transmission system (i.e. as a light pipe) and / or as ducting for cooling air (i.e. as a cooling and ventilation system). Investigation of possible solutions continues.

\section{References}

[1] H. A. Aberrahim, MYRRHA project status and contribution for HLW management and SMR based on Lead Technology, 4th Workshop on ADS and thorium, Proceedings of Science 2016. .

[2] Y. He, The present status and future plans of Chinese ADS project, 4th Workshop on ADS and thorium, Proceedings of Science 2016

[3] R. Garoby, Status and challenges of the European Spallation Source, 4th Workshop on ADS and thorium, Proceedings of Science 2016.

[4] M. McCarthy et al., A method for cooling RF waveguides on APT, Proceedings of the 1999 Particle Accelerator Conference, New York, 1999.

[5] R. E. May, Characteristics and effects of CW high power breakdown in waveguide, IEEE-MTT-S International Microwave Symposium 151-153 1976

[6] A. S. Acampora, P. T. Sproul,Waveguide breakdown effects at high average power and long pulse length, The Bell Syst. Tech. J., 51(9):2065-2091 1972.

[7] J. Lekner, Near approach of two conducting spheres: Enhancement of external electric field, J. of Electrost. 69: 559-563 2011.

[8] I. A. Krause Waveguide arc detector, US Patent 3,548,260 1970

[9] D. Valuch et al., Performance of arc detector of LHC high power RF system, Proceedings of IPAC2011 TUPS072 2011

[10] S. Schröder et al., Modeling of light scattering in different regimes of surface roughness. Optics Express 19(10):9821-9835 2011. 